\title{
A Novel Adaptive Neural Network Constrained Control for Multi-area Interconnected Power System with Hybrid Energy Storage
}

\author{
Dezhi Xu, Member, IEEE, Jianxing Liu, Member, IEEE, Xing-Gang Yan, and Wenxu Yan
}

\begin{abstract}
This paper concentrates on the problem of control of a hybrid energy storage system (HESS) for an improved and optimized operation of load-frequency control (LFC) applications. The HESS consists of a supercapacitor served as the main power source, and a fuel cell served as the auxiliary power source. Firstly, a Hammerstein-type neural network (HNN) is proposed to identify the HESS system, which formulates the Hammerstein model with a nonlinear static gain in cascade with a linear dynamic block. It provides the model information for the controller to achieve the adaptive performance. Secondly, a feedforward neural network based on back-propagation training algorithm is designed to formulate the PID-type neural network (PIDNN), which is used for the adaptive control of HESS system. Meanwhile, a dynamic anti-windup signal is designed to solve the operational constraint of the HESS system. Then, an appropriate power reference signal for HESS can be generated. Thirdly, the stability and the convergence of the whole system are proved based on the Lyapunov stability theory. Finally, simulation experiments are followed through on a four-area interconnected power system to demonstrate the effectiveness of the proposed control scheme.
\end{abstract}

Index Terms-Load-frequency control (LFC), hybrid energy storage system (HESS), Hammerstein network identification, PIDtype neural network (PIDNN), dynamic anti-windup, adaptive control

\section{INTRODUCTION}

$\mathbf{F}$ REQUENCY stability is an important index of power quality in power system. Any sudden changes of load may lead to the changes of the tie-line power and the fluctuation of the system frequency. Load frequency control (LFC) is one of the main measures to guarantee the power quality. The purpose of the LFC is to maintain the system frequency at the nominal value and minimize the tie power between different control areas [1-9]. However, LFC can not achieve sufficient control performance even for small load disturbance, while

Manuscript received ; revised.

Copyright (C)2015 IEEE. Personal use of this material is permitted. However, permission to use this material for any other purposes must be obtained from the IEEE by sending a request to pubspermissions@ieee.org.(Corresponding author: D. Xu)

This work was partially supported by the National Natural Science Foundation of China $(61503156,61403161,61473250)$ and National Key Research and Development Program (2016YFD0400300).

D. $\mathrm{Xu}$ and W. Yan are with Institute of Automation, Jiangnan University, Wuxi, Jiangsu 214122, China (e-mail: xudezhi@jiangnan.edu.cn, lutxdz@126.com)

J. Liu is with the Research Institute of Intelligent Control and Systems, Harbin Institute of Technology, Harbin 150001, China (e-mail: jx.liu@hit.edu.cn).

X.-G. Yan is with School of Engineering and Digital Arts, University of Kent, Canterbury, CT2 7NT, U.K. (e-mail: x.yan@kent.ac.uk). the system nonlinearity such as the generation rate constraint and dead band of governor are considered, which may lead to unsatisfactory frequency deviations and tie power oscillation [1-2]. System oscillation may be expanded in the system and even causes the power outages. The supplement of a smallcapacity energy storage system to power system can effectively promote the situation above [1-3]. Different types of advanced energy storage technologies can be applied in LFC, such as battery energy storage system [10], electrochemical energy storage [11], superconducting magnetic energy storage [1214], supercapacitor energy storage $[1,3,15]$, pumped storage [16], and flywheel energy storage [17].

Among the above technologies, the fuel cell has the characteristics of low operating temperature, quick start, good stability, no radiation and air pollution [18-20]. However, the dynamic response of fuel cell has a certain delay. An energy management unit with an energy storage device is required to improve the dynamic performance of the fuel cell generally, reduce the damage caused by rapid load change and prolong the service life [21]. Supercapacitor has the high power density, and its energy can be quickly released and stored [22, 23]. The lifetime of supercapacitor is measured in decades with no life degradation owing to the frequent cycling [1]. The application of a small-capacity HESS composed of fuel cell and supercapacitor to an interconnected power system for promoted LFC is an effective method, in which an inner control is used to exchange the desired power between the hybrid energy storage system (HESS) and the power system for improving the dynamic characteristics of LFC. The main controller objective is to regulate the frequency and tie-power. But the voltage of HESS must obtain its nominal value after dealing with a load disturbance for constant control [24].

In addition, the constraints and the restrictions of the smallrating HESS limited by its converter need to be considered [25]. In other words, the problem of control input limitations always exists in practical systems. The control signals are generally required to generate power through the actuator to drive the controlled system. However, its output amplitude can not be infinitely enlarged due to the physical limitation, which is called input saturation problem [26-27]. If this limitation is not taken into consideration, the characteristic of saturation may lead to undesired behavior even instability in closed loop system. To solve this nonlinear problem of input saturation of controller, the anti-windup compensation design method has been widely used [18, 26-30]. However, a large number of research results are focused on continuous-time systems 
instead of discrete-time systems.

Power systems are complicated multi-input multi-output high-order systems. Most advanced control methods can not be applied to the actual system due to the lack of accurate mathematical model. Therefore, system identification has become one of the core contents of the control system, which has received more and more attention in the power generation system [31]. For identification of nonlinear systems, Hammerstein and Wiener models have proposed the popular method for system modeling [32-37]. The Hammerstein model consists of a nonlinear static block in cascade with a linear dynamic block, while the Wiener model is composed of the same blocks but in the reverse order [32]. In order to obtain appropriate performance and adaptive ability, neural networks were used in the Hammerstein and wiener models to identify the parameters online for every sampling period [32-34].

For the LFC in power system, the conventional PID controller is one of popular control method widely used in engineering practice, because of the advantages of the simple and convenient structure, and no need for detailed information about the system dynamics [38]. However, it is very difficult to determine the satisfactory parameters of PID control in the presence various nonlinearities and uncertainties, which makes the dynamic performance of the PID controller to be relatively poor with the large overshoot, long setting time and oscillation with the transient frequency. In addition, the PID controller does not have any optimization calculation. Thus it does not have capability of frequency modulation unit fully in the frequency modulation process. As the development of advanced control algorithms, neural network based control method has been used in industry applications successfully, such as PID neural network [32, 39] due to the characteristics of self-learning, self-adaptation and self-organizing. The PID neural network can automatically identify and adjust the parameters of controller system.

Motivated by aforementioned investigation, two type neural networks are proposed to design a Hammerstein model identifier and a PID controller, respectively. One is Hammersteintype neural network (HNN), which formulates the Hammerstein model with a nonlinear static gain in cascade with a linear dynamic block. The other one is a PID-type neural network (PIDNN) that formulates a conventional PID controller. The HNN is designed to learn the power system with the HESS from the input-output data, which provides the model information online to update the parameters of the PIDNN controller, so that the controller has the abilities of self-learning and selfadaptation. Then, the PIDNN controller supplies the desired and appropriate power flow reference for the HESS to improve unsatisfactory frequency deviations and tie power oscillation. Moreover, considering the capacity of HESS, a dynamic antiwindup signal is designed to solve the operational constraints of HESS. Furthermore, the stability analysis of the whole closed-loop system is presented for the HNN and PIDNN controllers by Lyapunov theory. The simulation results demonstrate the effectiveness of the proposed control scheme, which reduces the frequency and tie-power deviations tremendously compared with the conventional PID controller with HESS and the LFC without HESS. The proposed control scheme can be applied to other mechanical and electrical systems.

\section{HESS FOR MULTI-AREA INTERCONNECTED POWER SYSTEM}

In this paper, LFC of four-area interconnected power system with HESS is taken as the research object. To demonstrate the effectiveness of the designed control scheme, a four area power system model with governor and reheating steam turbine is developed, which is shown in Fig. 1.

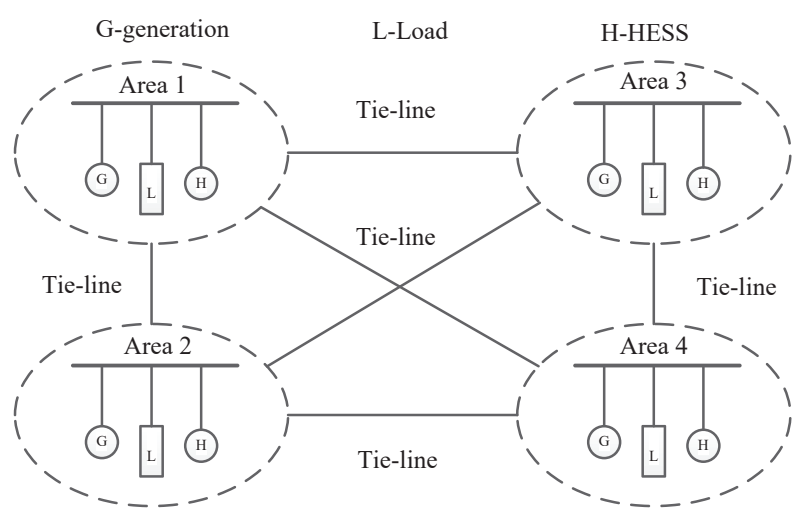

Fig. 1. Schematic of the four-area interconnected power system with HESS.

\section{A. Model of Load Frequency Control}

Power system is a complex nonlinear dynamic system, because the power system only subjects to small load interference under normal operating conditions. The common method to achieve the system model is linearization around the operating point. Then the dynamic model of the power system can be represented by the linearized model near the operating point. An improved $I$ th $(I=1,2,3,4)$ control area of interconnected power system is shown in Fig. 2 [1]. The linearized model includes governor, reheating steam turbine and generator. Each part of the model in Fig. 2 is expressed as follows:

The model of governor is expressed as follows:

$$
G_{g I}=\frac{1}{T_{g I} s+1}
$$

The model of generator is expressed by:

$$
G_{p I}=\frac{K_{p I}}{T_{p I} s+1}
$$

The model of reheating steam turbine and generator is expressed by:

$$
G_{t I}=\frac{c T_{r I} s+1}{\left(T_{r I} s+1\right)\left(T_{T I} s+1\right)}
$$

where $T_{g I}$ is time constant of governor, $T_{p I}$ is time constant of generator, $K_{p I}$ is generator gain, $T_{r I}$ is time constant of reheating device, $T_{T I}$ is turbine time constant, $c$ is the gain of reheating device, $R_{I}$ is droop characteristic, $u_{l f c I}$ is the control variable of LFC, $\Delta P_{d I}$ is the load disturbance, $T_{I J}$ is the interconnection constant between the control $I$ and control area $J \neq I,(J=1,2,3,4), K_{A F I}$ is the proportional 


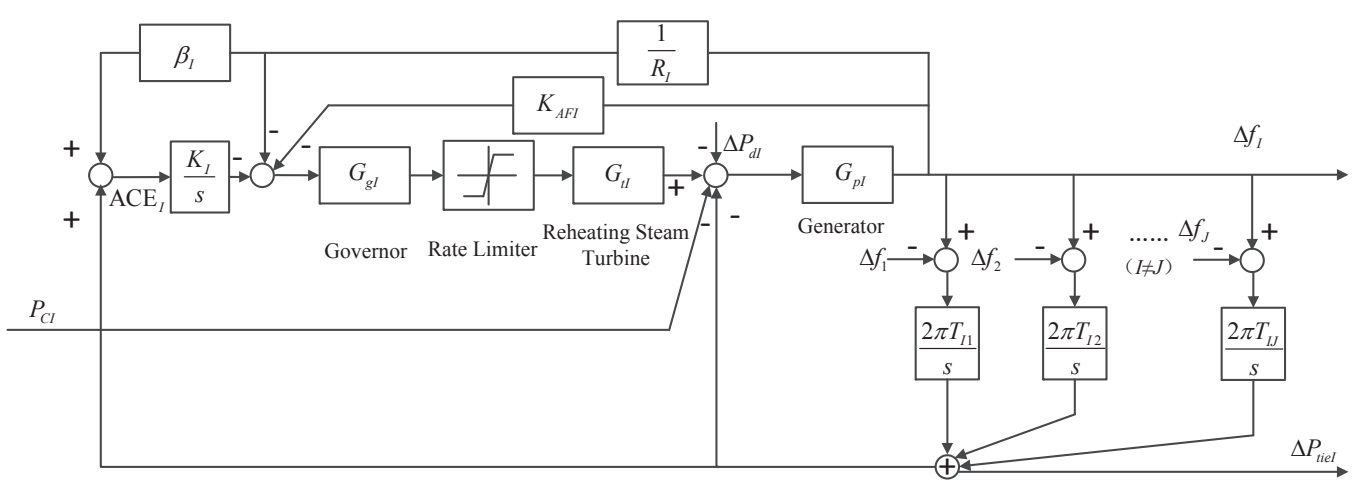

Fig. 2. Simulation model of $i$ th control area of interconnected power system.

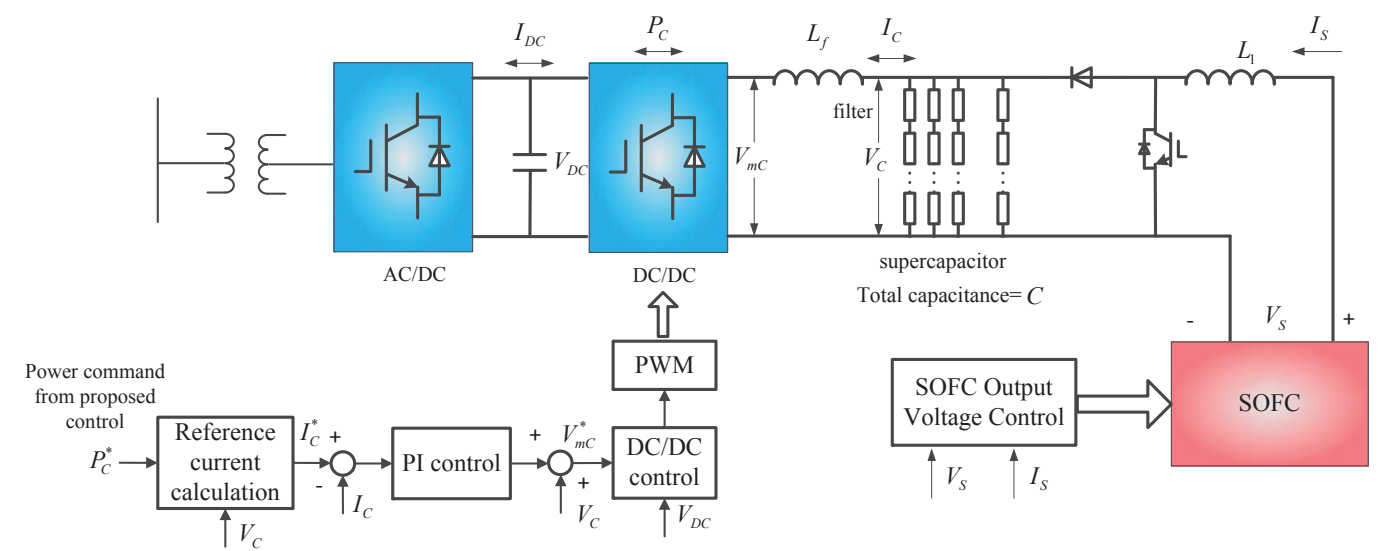

Fig. 3. Basic structure of HESS.

negative feedback coefficient of frequency deviation which is used to improve the system performance [1], $P_{C I}$ is the power delivered by HESS to the control area [3].

The aim of load frequency control of multi-area interconnected power system is to ensures not only the frequency deviation of each region to return to zero, but also to adjust the interconnected power of tie-line to the desired value. Therefore, load frequency control of multi-area power system adopts area control error (ACE) as feedback variable to meet the above two control objectives. The ACE of Ith control area is defined as following:

$$
\mathrm{ACE}_{I}=\Delta P_{t i e I}+\psi_{I} \Delta f_{I}
$$

where $\Delta f_{I}$ is the frequency deviation, $\Delta P_{\text {tieI }}$ is the interchanged power of tie-line, $\psi_{I}$ is the frequency deviation constant.

Then the feedback control form can be expressed as:

$$
u_{l f c I}=-\frac{K_{I}}{s} \mathrm{ACE}_{I}
$$

where $\mathrm{ACE}_{I}$ is the area control error of $I$ th control area, $K_{I}$ is control constant of $I$ th control area.

\section{B. Hybrid Energy Storage System}

The details of the power conversion system applied for interfacing the HESS are shown in Fig. 3. The control strategy of the AC/DC converter is to stabilize the DC-link voltage and connect the HESS system. The control strategy of the $\mathrm{DC} / \mathrm{DC}$ converter is to realize the bidirectional power flow between the power grid and the HESS according to the given reference power signal. The control strategy of the above two converters is similar to the work in [3]. In addition, fuel cell serving as auxiliary power source is used to supply energy for supercapacitor by the boost circuit when the energy levels of supercapacitor exceed its normal range.

The HESS topology used in our paper has the following advantages: 1) Fuel cell runs without pollution and the only product of the process is water; 2) Its short-time overload capacity can reach $200 \%$ rated power; 3 ) The power generation efficiency of fuel cells is high, and it directly converts chemical energy into electrical energy without the restriction of the Carnot cycle; 4) A wide range of fuel types. Fuel cells are powered by hydrogen and oxygen, where the oxygen can be directly obtained from the air, and the fuel hydrogen can be made from fossil fuels such as natural gas, methanol, ethanol (alcohol), oil and coal; 5) Ultracapacitor has the characteristics of large power density, short charge and discharge time, high reliability and long cycle life.

The HESS interfaced with four-area interconnected power system respectively, are used to restrain the electromechanical oscillations, control frequency and interchanged power of tie-line deviation to zero. The proposed controller of this paper needs to command the HESS system to provide a fast power response when a load perturbation occurs in each 
interconnected power system.

1) Mode of Supercapacitor: Supercapacitor is a new energy storage unit with high power density, large capacity and many cycles. The supercapacitor of HESS applied in this paper is composed of a number of Maxwell Boostcap modules BMOD0018-P390 (18F/390V) yielding a structure constituting a structure of $6 \mathrm{~F} / 1170 \mathrm{~V} 750 \mathrm{~kW}[3,15]$. The DCbus voltage of line-side converter is assumed to maintain to at $2.5 \mathrm{kV}$. The nominal voltage of supercapacitor $V_{C}$ is chosen to be $925 \mathrm{~V}$. In addition, the supercapacitor is operating at the center energy levels of maximum $1170 \mathrm{~V}$ and minimum $585 \mathrm{~V}$.

As given in Fig. 3, the PI controller converts the power command received from proposed controller into a current command for the DC/DC converter to control the energy of supercapacitor, to achieve the desired current tracking. The following expression gives the average output voltage $V_{S C}$ of DC/DC converter in terms of DC-link voltage $V_{D C}$ [3]:

$$
V_{S C}=m V_{D C}
$$

The modulation coefficient $m \in[0,1]$ is defined as:

$$
m= \begin{cases}D, & \text { buck mode } \\ 1-D, & \text { boost mode }\end{cases}
$$

where $D$ represents the duty cycle of the buck/boost switch, which depends on the operation mode.

2) Mode of Fuel Cell: The dynamic response of fuel cell has a little delay, because the inner part of the fuel cell is an electrochemical reaction. Sudden changes in load will lead to exceeding standard of some indicators and affecting their service life. So an energy management unit with an energy storage device is required to improve the dynamic performance of the fuel cell and to reduce the damage caused by rapid load change and prolong the service life.

In this paper, solid oxide fuel cells (SOFC) serving as auxiliary power source is used to supply energy. The SOFC dynamics model has been widely accepted widely is used as the research model [18-19]. An improved data driven model-free adaptive constrained control [18] has been used to maintain the output voltage of SOFC $V_{S}$ at $333 \mathrm{~V}$. Boost circuit applied for SOFC is used to provide energy to the supercapacitor and keep its voltage $V_{C}$ at rated voltage when the voltage of the supercapacitor drops without taking the energy management strategy into consideration.

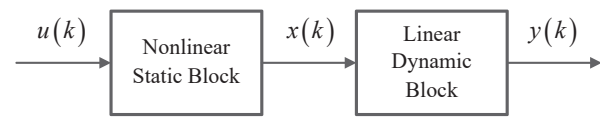

Fig. 4. Hammerstein model.

In next section, denote the $I$ th area control error by $\mathrm{ACE}_{I}$, and define the $I$ th power command $P_{C I}^{*}$ of supercapacitor as the control objective $y$ and control input $u$, respectively.

\section{HNN IDENTIFICATION FOR LFC OF THE ITH AREA INTERCONNECTED POWER SYSTEM}

Hammerstein model shown in Fig. 4 is a nonlinear system consisted of a nonlinear static gain in cascade with a linear dynamic block, which can approximate nonlinear function effectively.

\section{A. Hammerstein Model Formulation}

If the nonlinear static block is assumed to be approached by a finite polynomial expansion, the autoregressive Hammerstein model for LFC of multi-area interconnected power system with HESS can be expressed in the following form [36]:

$$
\alpha(z) y(k)=\beta(z) x(k)
$$

where

$$
\left\{\begin{array}{l}
x(k)=\gamma_{1} u(k)+\gamma_{2} u^{2}(k)+\cdots+\gamma_{n_{\gamma}} u^{n_{\gamma}}(k) \\
\alpha(z)=1+\alpha_{1} z^{-1}+\alpha_{2} z^{-2}+\cdots+\alpha_{n_{\alpha}} z^{-n_{\alpha}} \\
\beta(z)=\beta_{0}+\beta_{1} z^{-1}+\beta_{2} z^{-2}+\cdots+\beta_{n_{\beta}} z^{-n_{\beta}}
\end{array}\right.
$$

where $x(k)$ represents the intermediate variable, $n_{\gamma}$ determines the order of nonlinear static block, $n_{\alpha}$ and $n_{\beta}$ determines the order of linear dynamic block of Hammerstein model, and $n_{\beta} \leq n_{\alpha} . z^{-1}$ represents the unit delay operator. $\alpha_{i},\left(i=1,2, \cdots, n_{\alpha}\right)$ and $\beta_{j},\left(j=1,2, \cdots, n_{\beta}\right)$ are linear dynamic block parameters of Hammerstein model, and $\gamma_{l},\left(l=1,2, \cdots, n_{\gamma}\right)$ are nonlinear static block parameters of Hammerstein model.

The intermediate variable $x(k)$ is non-measurable and has no physical meaning, which can be eliminated by equations (9). Substituting (9) into (8), the expression in operator form is obtained as follows:

$$
y(k)=\frac{\beta(z)}{\alpha(z)} \sum_{l=1}^{n_{\gamma}} \gamma_{l} u^{l}(k)
$$

\section{B. Hammerstein-type Neural Network Identification}

The structure of HNN is designed and shown in Fig. 5, which is composed of a single dynamic node with two tapped delay lines, and these delay lines form the nonlinear static element and linear dynamic element.

Therefore, the output $\hat{y}(k)$ of HNN can be expressed as:

$$
\begin{aligned}
\hat{y}(k)= & -\hat{\alpha}_{1} \hat{y}(k-1)-\hat{\alpha}_{2} \hat{y}(k-2)-\cdots-\hat{\alpha}_{n_{\alpha}} \hat{y}\left(k-n_{\alpha}\right) \\
& +\hat{\beta}_{0} \hat{x}(k)+\hat{\beta}_{1} \hat{x}(k-1)+\cdots+\hat{\beta}_{n_{\beta}} \hat{x}\left(k-n_{\beta}\right) \\
= & -\sum_{i=1}^{n_{\alpha}} \hat{\alpha}_{i} \hat{y}(k-i)+\sum_{j=0}^{n_{\beta}} \hat{\beta}_{j} \hat{x}(k-j)
\end{aligned}
$$

The hidden layer $\hat{x}(k)$ of HNN can be described as:

$$
\hat{x}(k)=\sum_{l=1}^{n_{\gamma}} \hat{\gamma}_{l} u^{l}(k)
$$

where the parameters $\hat{\alpha}_{i},\left(i=1,2, \cdots, n_{\alpha}\right), \hat{\beta}_{j},(j=$ $\left.1,2, \cdots, n_{\beta}\right)$ and $\hat{\gamma}_{l}\left(l=1,2, \cdots, n_{\gamma}\right)$ of HNN are associated weights in (8) and (9), respectively. In this section, negative gradient training method applied for adjusting the weights of HNN is executed to identify the Hammerstein model. The identification error can be described as follows:

$$
J_{H_{1}}(k)=\frac{1}{2}(y(k)-\hat{y}(k))^{2}=\frac{1}{2} \hat{e}^{2}(k)
$$



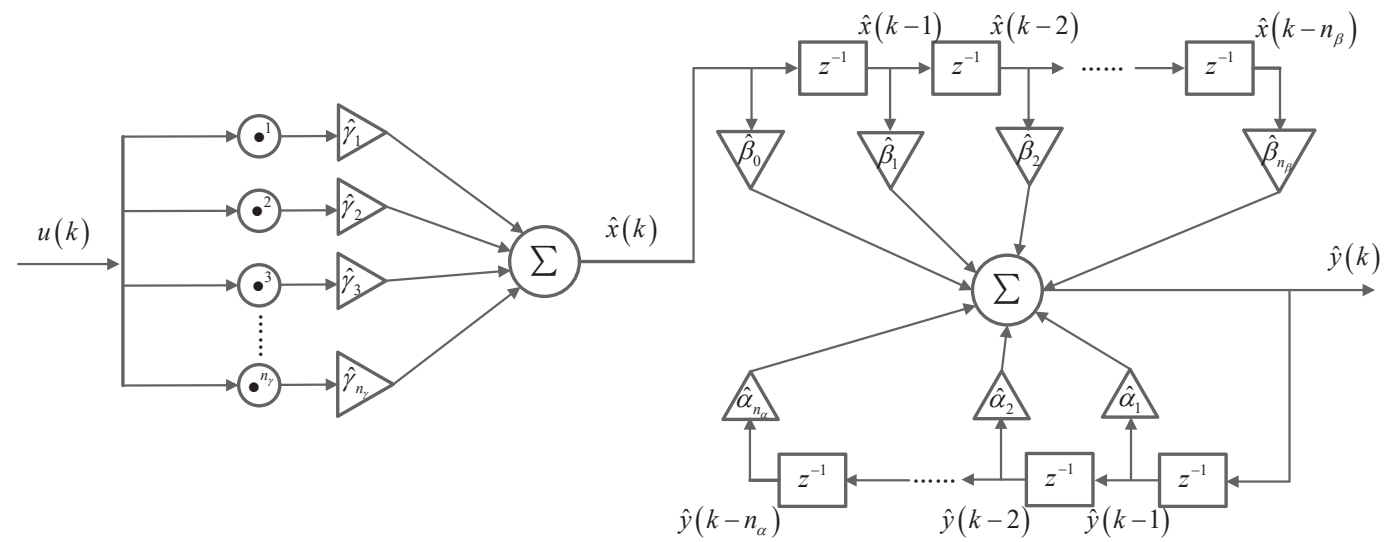

Fig. 5. The structure of Hammerstein-type neural network.

where $\hat{e}(k)=y(k)-\hat{y}(k)$, parameter $H_{1}$ represents the weights $\hat{\alpha}_{i},\left(i=1,2, \cdots, n_{\alpha}\right), \hat{\beta}_{j},\left(j=1,2, \cdots, n_{\beta}\right)$ and $\hat{\gamma}_{l},\left(l=1,2, \cdots, n_{\gamma}\right)$. The error function (13) needs to be achieved and minimized with respect to the parameter $H_{1}$ of the HNN by the BP training algorithm. The partial derivative of (13) can be calculated as:

$$
\frac{\partial J_{H_{1}}(k)}{\partial H_{1}(k)}=-\hat{e}(k) \frac{\partial \hat{y}(k)}{\partial H_{1}(k)}
$$

The general update rule of the point can be expressed as:

$$
\begin{aligned}
H_{1}(k+1) & =H_{1}(k)+\Delta H_{1}(k)=H_{1}(k)-\eta_{H} \frac{\partial J_{H_{1}}(k)}{\partial H_{1}(k)} \\
& =H_{1}(k)+\eta_{H} \hat{e}(k) \frac{\partial \hat{y}(k)}{\partial H_{1}(k)}
\end{aligned}
$$

Where $\eta_{H}$ represents the training rate of the weights of HNN.

According to (11) and (12), the partial derivative of $\hat{y}(k)$ about $\hat{\alpha}_{i},\left(i=1,2, \cdots, n_{\alpha}\right), \hat{\beta}_{j},\left(j=1,2, \cdots, n_{\beta}\right)$, and $\hat{x}(k)$ can be calculated as follows:

$$
\begin{gathered}
\frac{\partial \hat{y}(k)}{\partial \hat{\alpha}_{i}}=-\hat{y}(k-i), \frac{\partial \hat{y}(k)}{\partial \hat{\beta}_{j}}=\hat{x}(k-j) \\
\frac{\partial \hat{y}(k)}{\partial \hat{x}(k)}=\hat{\beta}_{0}
\end{gathered}
$$

Combining (11) and (12) with (17), the partial derivative of $\hat{y}(k)$ to $\hat{\gamma}_{l},\left(l=1,2, \cdots, n_{\gamma}\right)$ can be computed as following:

$$
\frac{\partial \hat{y}(k)}{\partial \hat{\gamma}_{l}}=\frac{\partial \hat{y}(k)}{\partial \hat{x}(k)} \frac{\partial \hat{x}(k)}{\partial \hat{\gamma}_{l}}=\hat{\beta}_{0} u^{l}(k)
$$

From (15), (16) and (18), the update law of the weights $\hat{\alpha}_{i},\left(i=1,2, \cdots, n_{\alpha}\right), \hat{\beta}_{j},\left(j=1,2, \cdots, n_{\beta}\right)$ and $\hat{\gamma}_{l},(l=$ $\left.1,2, \cdots, n_{\gamma}\right)$ of HNN can be described as:

$$
\begin{aligned}
& \hat{\alpha}_{i}(k+1)=\hat{\alpha}_{i}(k)+\eta_{H} \hat{e}(k) \frac{\partial \hat{y}(k)}{\partial \hat{\alpha}_{i}} \\
& \hat{\beta}_{j}(k+1)=\hat{\beta}_{j}(k)+\eta_{H} \hat{e}(k) \frac{\partial \hat{y}(k)}{\partial \hat{\beta}_{j}} \\
& \hat{\gamma}_{l}(k+1)=\hat{\gamma}_{l}(k)+\eta_{H} \hat{e}(k) \frac{\partial \hat{y}(k)}{\partial \hat{\gamma}_{l}}
\end{aligned}
$$

Therefore, the output $\hat{y}(k)$ of HNN can be determined by these update laws, and the weights $\hat{\alpha}_{i},\left(i=1,2, \cdots, n_{\alpha}\right)$, $\hat{\beta}_{j},\left(j=1,2, \cdots, n_{\beta}\right)$ and $\hat{\gamma}_{l},\left(l=1,2, \cdots, n_{\gamma}\right)$ can be adjusted, simultaneously. Then, according to the analysis above, the convergence criterion for selecting appropriate learning rate is proposed as follows:

Theorem 1: If $\eta_{H}$ is selected satifying

$$
0<\eta_{H}<\frac{2}{\Phi_{H}^{2}}
$$

where $\Phi_{H}=\max _{k}\left\|\frac{\partial \hat{y}(k)}{\partial H_{1}}\right\|$, then, the modeling error $\hat{e}(k)$ is converged to the origin asymptotically by the update laws (19).

Proof: Consider a candidate Lyapunov function

$$
V_{H}(k)=\frac{1}{2} \hat{e}^{2}(k)
$$

Then, according to (15), it follows that

$$
\Delta H_{1}(k)==H_{1}(k+1)-H_{1}(k)=\eta_{H} \hat{e}(k) \frac{\partial \hat{y}(k)}{\partial H_{1}(k)}
$$

From (20)-(22), $\Delta V_{H}(k)$ can be computed as:

$$
\begin{aligned}
& \Delta V_{H}(k)=V_{H}(k+1)-V_{H}(k) \\
& =\Delta \hat{e}(k)\left(\hat{e}(k)+\frac{\Delta \hat{e}(k)}{2}\right) \\
& =-\eta_{H} \hat{e}^{2}(k)\left\|\frac{\partial \hat{y}(k)}{\partial H_{1}(k)}\right\|^{2}+\frac{1}{2} \eta_{H}^{2} \hat{e}^{2}(k)\left\|\frac{\partial \hat{y}(k)}{\partial H_{1}(k)}\right\|^{4} \\
& \leq-\frac{1}{2} \eta_{H} \hat{e}^{2}(k)\left(2-\eta_{H} \Phi_{H}^{2}\right)\left\|\frac{\partial \hat{y}(k)}{\partial H_{1}(k)}\right\|^{2}
\end{aligned}
$$

From the fact that $V_{H}(k)$ is positive definite, $V_{H}(k) \geqslant 0$ for all of $k, \Delta V_{H}(k)$ is negative definite when the $\eta_{H}$ satisfies (20). Therefore, it follows that $\lim _{k \rightarrow \infty} \hat{e}(k)=0$.

Generally, $y(k) \approx \hat{y}(k)$, since HNN is trained online. Therefore, according to (12) and (17), Jacobian information (system sensitivity) can be computed as:

$$
\begin{aligned}
\frac{\partial y(k)}{\partial u(k)} & \approx \frac{\partial \hat{y}(k)}{\partial u(k)}=\frac{\partial \hat{y}(k)}{\partial \hat{x}(k)} \frac{\partial \hat{x}(k)}{\partial u(k)} \\
& =\hat{\beta}_{0} \sum_{l=1}^{n_{\gamma}} l \cdot \hat{\gamma}_{l} u^{l-1}(k), \quad l=1,2, \cdots, n_{\gamma}
\end{aligned}
$$




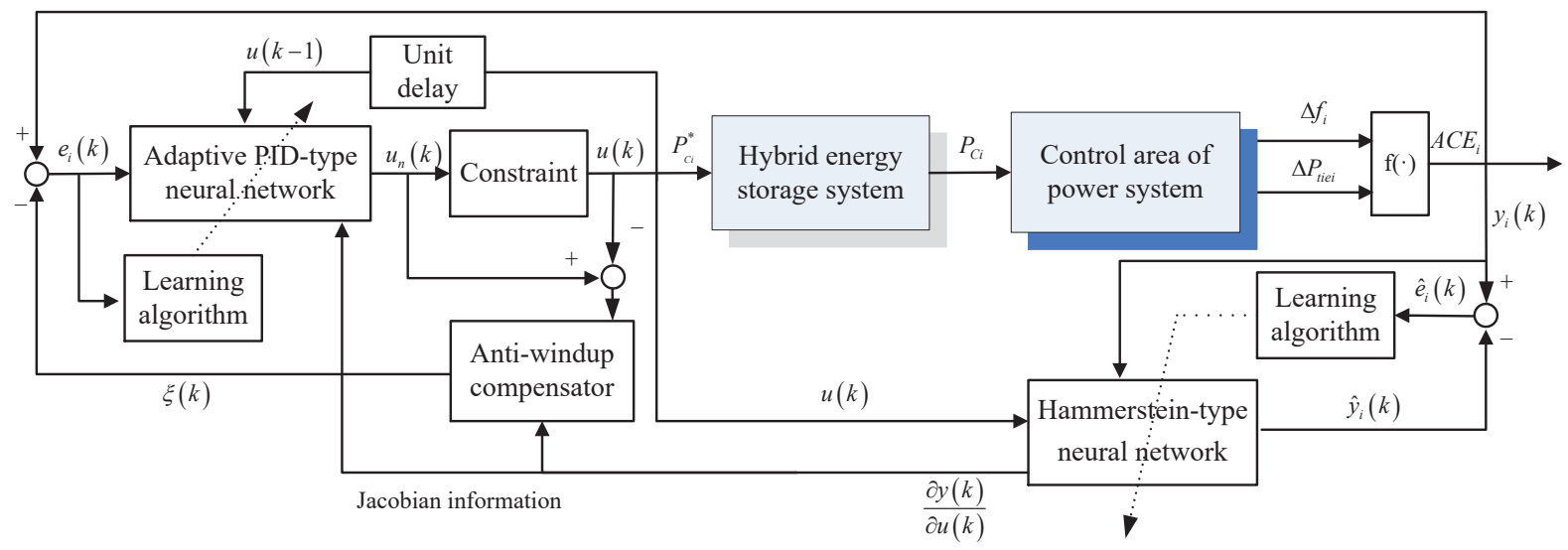

Fig. 6. Adaptive PIDNN control for $i$ th control area of interconnected power system based on HNN.

\section{ADAPTIVE PIDNN CONSTRAINED CONTROLLER BASED ON HNN}

In this section, the control objective is to adjust the parameters of the PIDNN controller to supply the appropriate power reference signal to the HESS so that the output $y(k)$ of LFC can track the desired reference signal $y^{*}(k)$. The signal $y^{*}(k)$ is set as zero, which can adjust the frequency deviation $\Delta f_{I}$ and the interchanged power of tie-line power $\Delta P_{t i e I}$ to zero. In addition, the weights of HNN is tuning online so that the $\hat{y}(k)$ can approach the system output $y(k)$ in real time, which also provides the Jacobian information to parameters tuning of the PIDNN controller. The whole control scheme for control area $I,(I=1,2,3,4)$ is depicted in Fig. 6. In this picture, the block labeled as "Hybrid energy storage system" represents the model of HESS shown in Fig. 3 and the block labeled as "Control area of power system" represents the simulation model of the $I$ th control area of the interconnected power system depicted in Fig. 2 [3].

Firstly, the tracking error can be defined as:

$$
e(k)=y^{*}(k)-y(k)-\xi(k)
$$

where reference signal $y^{*}(k)$ is zero, $\xi(k)$ represents a compensation signal which is used to adjust the reference setting value to maintain the control input $u(k)$ within the saturation range, which is designed as:

$$
\xi(k)=\rho \xi(k-1)+\frac{\partial y(k)}{\partial u(k)}\left(u_{n}(k)-u(k)\right)
$$

where $\rho<1$ is selected within the unit circle.

Since the rated capacity of converter of HESS is $1 \%$ of area capacity, then the $u_{\max }=0.01 \mathrm{p} . \mathrm{u}$. and $u_{\min }=-0.01 \mathrm{p} . \mathrm{u}$., so the control signal $u(k)$ can be defined as following:

$$
u(k)=\operatorname{Sat}\left(u_{n}(k)\right)= \begin{cases}u_{\max }, & u_{n} \geq u_{\max } \\ u_{n}, & u_{\min }<u_{n}<u_{\max } \\ u_{\min }, & u_{n} \leq u_{\min }\end{cases}
$$

where $u_{\min }$ and $u_{\max }$ are the lower and upper bounds of the Sat $(\cdot)$ function, respectively.
Using the identification result of HNN, the modified PIDNN controller with the constraint in our paper is given as follows:

$$
u_{n}(k)=u(k-1)+\sum_{j=1}^{3} k_{j}(k) h\left(\text { net }_{j}\right)
$$

where $k_{j}(k), j=1,2,3$ represent the parameters of PIDNN controller, and the output of first layer net $_{j}$ is given by:

$$
\text { net }_{j}=\sum_{i=1}^{3} v_{i j} x_{c i}(k)
$$

where $v_{i j}, i=1,2,3$ and $j=1,2,3$ are the weights of input layer and hidden layer, respectively, and

$$
\begin{aligned}
& x_{c 1}=e(k)-e(k-1), x_{c 2}=e(k) \\
& x_{c 3}=e(k)-2 e(k-1)+e(k-2)
\end{aligned}
$$

In (28), $h(\cdot)$ is the nonlinear function of hidden layer, which is chosen as:

$$
h\left(\text { net }_{j}\right)=\frac{1-\exp ^{-n e t_{j}}}{1+\exp ^{-n e t_{j}}}, j=1,2,3
$$

The derivative of (30) can be calculated as:

$$
h^{\prime}\left(\text { net }_{j}\right)=\frac{1}{2}\left[1+h\left(\text { net }_{j}\right)\right]^{2}, j=1,2,3
$$

Then, in order to get the updated law for adaptive PIDNN controller, the error function is given as follows:

$$
J_{H_{2}}=\frac{1}{2} e^{2}(k)=\frac{1}{2}\left(y^{*}(k)-y(k)-\xi(k)\right)^{2}
$$

According to (32), the gradient of $J_{H_{2}}$ about the weights $k_{j}(k)$ and $v_{i j}(k)$ for $i=1,2,3$ and $j=1,2,3$ of the PIDNN can be calculated as:

$$
\begin{aligned}
& \frac{\partial J_{H_{2}}(k)}{\partial k_{j}(k)}=-e(k) \frac{\partial y(k)}{\partial u(k)} h\left(\text { net }_{j}\right) \\
& \frac{\partial J_{H_{2}}(k)}{\partial v_{i j}(k)}=-e(k) \frac{\partial y(k)}{\partial u(k)} k_{j}(k) h^{\prime}\left(\text { net }_{j}\right) x_{c i}(k)
\end{aligned}
$$

Therefore, according to (33), the general update rule of the weights in this point can be given as follows by use of the BP 

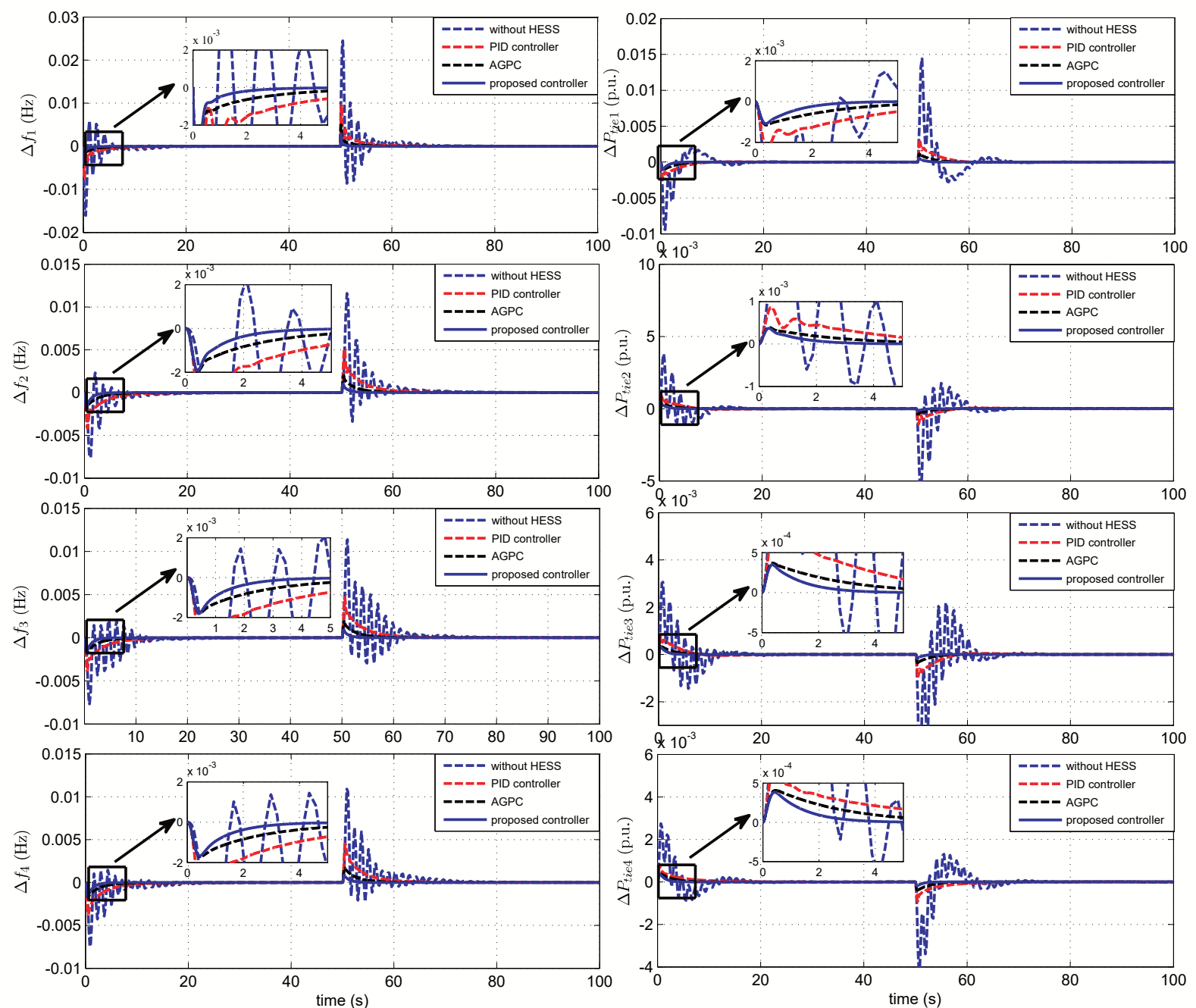

Fig. 7. System response of proposed controller compared with PID controller with HESS and the LFC without HESS for a load increment of 0.01 p.u. followed by a load rejection of 0.015 p.u.

training algorithm:

$$
\begin{aligned}
k_{j}(k+1) & =k_{j}(k)-\eta_{c} \frac{\partial J_{H_{2}}(k)}{\partial k_{j}(k)} \\
v_{i j}(k+1) & =v_{i j}(k)-\eta_{c} \frac{\partial J_{H_{2}}(k)}{\partial v_{i j}(k)}
\end{aligned}
$$

where $\eta_{c}$ is the learning rate of the PIDNN controller.

From the general update rule (36) and (37) of the weights, it follows that

$$
\Delta H_{2}(k)=\eta_{c} e(k) \frac{\partial e(k)}{\partial H_{2}(k)} \approx \eta_{c} e(k) \frac{\partial y(k)}{\partial u(k)} \frac{\partial u_{n}(k)}{\partial H_{2}(k)}
$$

where $H_{2}$ represents the weights $k_{j}(k)$ and $v_{i j}(k)$ for $i=$ $1,2,3$ and $j=1,2,3$ of PIDNN controller.

Similar to Theorem 1, the convergence criterion of PIDNN controller is necessary to be analyzed, because of the requirement of convergence speed and stability of controller. So, the criteria to choose an appropriate learning rate $\eta_{c}$ will be given as follows.

Theorem 2: If the $\eta_{c}$ is selected as

$$
0<\eta_{c}<\frac{2}{\Phi_{c 1}^{2} \Phi_{c 2}^{2}}
$$

where $\Phi_{c 1}=\max _{k}\left\|\frac{\partial y(k)}{\partial u(k)}\right\|, \Phi_{c 2}=\max _{k}\left\|\frac{\partial u_{c}(k)}{\partial H_{2}(k)}\right\|$ the system error $e(k)$ will converge to the origin asymptotically by using the update laws (34).

Proof: Consider the candidate Lyapunov function

$$
V_{c}(k)=\frac{1}{2} e^{2}(k)
$$

From (35)-(37)

$$
\begin{aligned}
& \Delta V_{c}(k)=V_{c}(k+1)-V_{c}(k) \\
& =\Delta e(k)\left(e(k)+\frac{\Delta e(k)}{2}\right) \\
& =\left(\frac{\partial e(k)}{\partial H_{2}(k)}\right)^{T} \Delta H_{2}(k)\left[e(k)+\frac{1}{2}\left(\frac{\partial e(k)}{\partial H_{2}(k)}\right)^{T} \Delta H_{2}(k)\right] \\
& \leq-\frac{1}{2} \eta_{c} e^{2}(k)\left(2-\eta_{c} \Phi_{c 1}^{2} \Phi_{c 2}^{2}\right)\left\|\frac{\partial y(k)}{\partial u(k)}\right\|^{2}\left\|\frac{\partial u_{n}(k)}{\partial H_{2}(k)}\right\|^{2}
\end{aligned}
$$

Similar as the proof of Theorem 1, it is concluded that the convergence of PIDNN can be guaranteed by use of (36). 
TABLE I

THE PARAMETERS OF FOUR-AREA INTERCONNECTED POWER SYSTEM

\begin{tabular}{cccccccccccc}
\hline Area & $T_{G}(\mathrm{~s})$ & $T_{R}(\mathrm{~s})$ & $T_{T}(\mathrm{~s})$ & $R[\mathrm{~Hz} /(\mathrm{p} . \mathrm{u} . \mathrm{Mw})]$ & $T_{I J}(\mathrm{~s})$ & $K_{p}[\mathrm{~Hz} /(\mathrm{p} . \mathrm{u} . \mathrm{Mw})]$ & $T_{P}(\mathrm{~s})$ & $c$ & $\beta[\mathrm{Hz} /(\mathrm{p} . \mathrm{u} . \mathrm{Mw})]$ & $K_{A F}$ \\
\hline 1 & 0.08 & 4.2 & 0.3 & 2.4 & 0.0707 & 120 & 20 & 0.35 & 0.425 \\
2 & 0.072 & 4.2 & 0.33 & 2.7 & 0.0707 & 112.5 & 25 & 0.35 & 0.425 \\
3 & 0.07 & 4.2 & 0.35 & 2.5 & 0.0707 & 125 & 20 & .35 & 0.425 \\
4 & 0.085 & 4.2 & 0.375 & 2 & 0.0707 & 115 & 1.1 & 1.1 \\
\hline
\end{tabular}
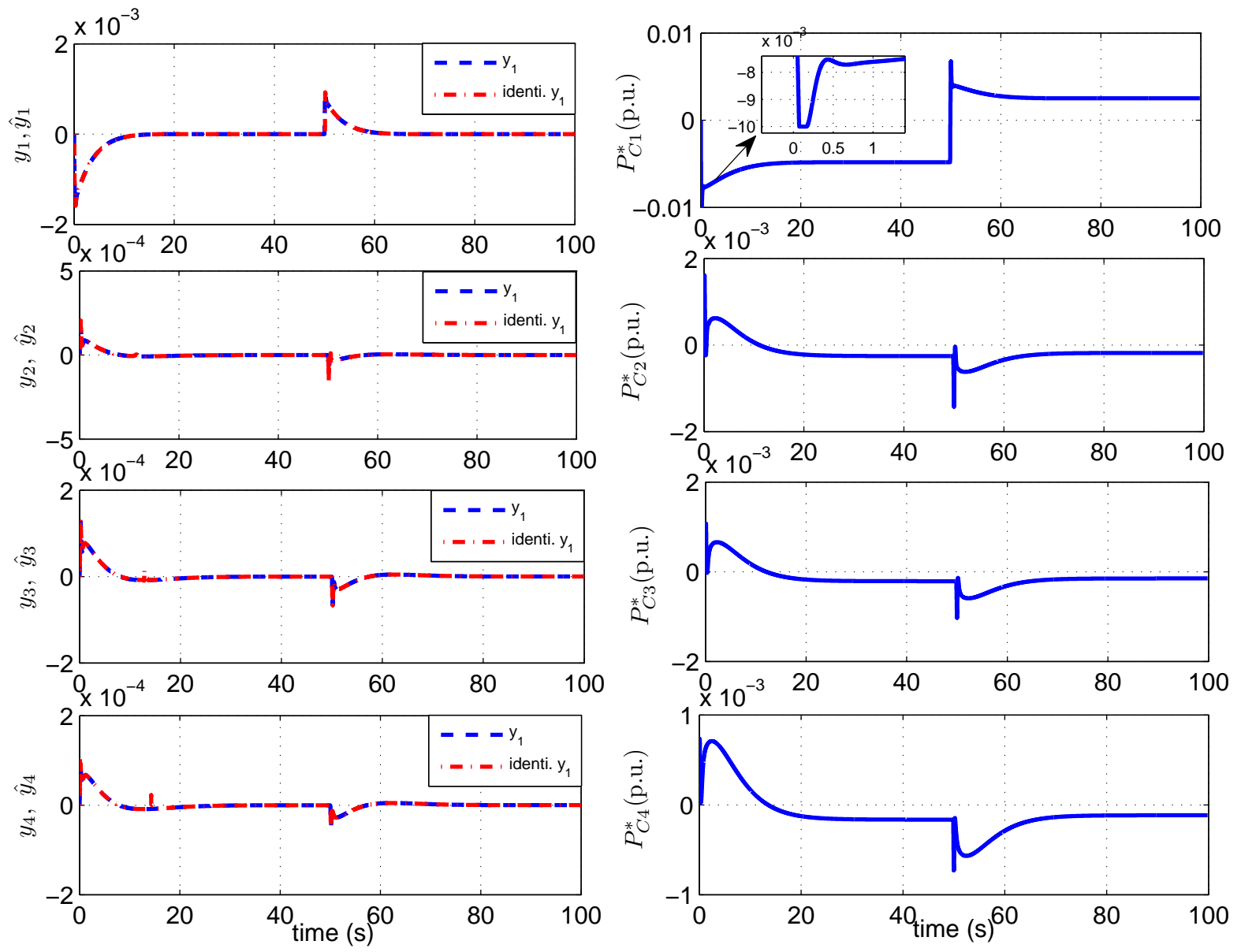

Fig. 8. Identification results of HNN and output of the PIDNN.

\section{Simulation Results}

In this section, the proposed adaptive constrained PIDNN controller based on HNN designed in Section IV is applied to four-area interconnected power system to obtain that the frequency deviation of each region returns to zero, and that also interconnected power of tie-line keeps at desired value. Meanwhile, it makes that the system of HESS satisfies the operating constraints under the condition of load disturbance.

In the simulation, the parameters of four-area interconnected power system are listed in Table. 1. The parameters of SOFC and its control parameter are shown in [18]. The capacity of the SOFC used in this paper is $200 \mathrm{~kW}$. The parameters of supercapacitor $C=6 F$ and filter inductor $L_{f}=1 \mathrm{mH}$ in HESS. The parameters of the PI controller in the DC/DC converter are $k_{p}=2$ and $k_{i}=1$. The sampling time is $T_{s}=0.001 \mathrm{~s}$ and the orders of $\mathrm{HNN}$ are selected as $n_{\alpha}=3, n_{\beta}=3, n_{\gamma}=3$. The learning rate of $\mathrm{HNN}$ for

four-area interconnected power system is chosen as $\eta_{H 1}=$ $\left[10^{4}, 10^{4}, 10^{4}\right]^{T}, \eta_{H 2}=\left[1.2 \times 10^{7}, 1.2 \times 10^{7}, 1.2 \times 10^{7}\right]^{T}$ and $\eta_{H 4}=\eta_{H 3}=\left[4 \times 10^{7}, 4 \times 10^{7}, 4 \times 10^{7}\right]^{T}$. The openloop input-output data can achieve the initial values of HNN for four-area interconnected power system.

For the proposed controller, the learning rate of $k_{j}$ and $v_{i j}$ are selected as $\eta_{c 1}=[1,1,1]^{T}$ and $\eta_{c 2}=[0.1,0.1,0.1]^{T}$, respectively. The initial values of PIDNN are the same as the parameters of traditional PID controller, which is chosen as $k_{1}(0)=5, k_{2}(0)=0.001$ and $k_{3}(0)=0.05$.

In the following case, the system is subjected to a load increment of $0.01 \mathrm{p} . \mathrm{u}(750 \mathrm{~kW})$ followed by a load rejection of 0.015p.u (1125kW). A higher load disturbance 0.015p.u. $(1125 \mathrm{~kW})$ is considered at time $50 \mathrm{~s}$, which is used to increase uncertainty of multi-area interconnected power system. The responses of the closed-loop system using the proposed controller are shown in Fig. 7. Meanwhile, to highlight the 

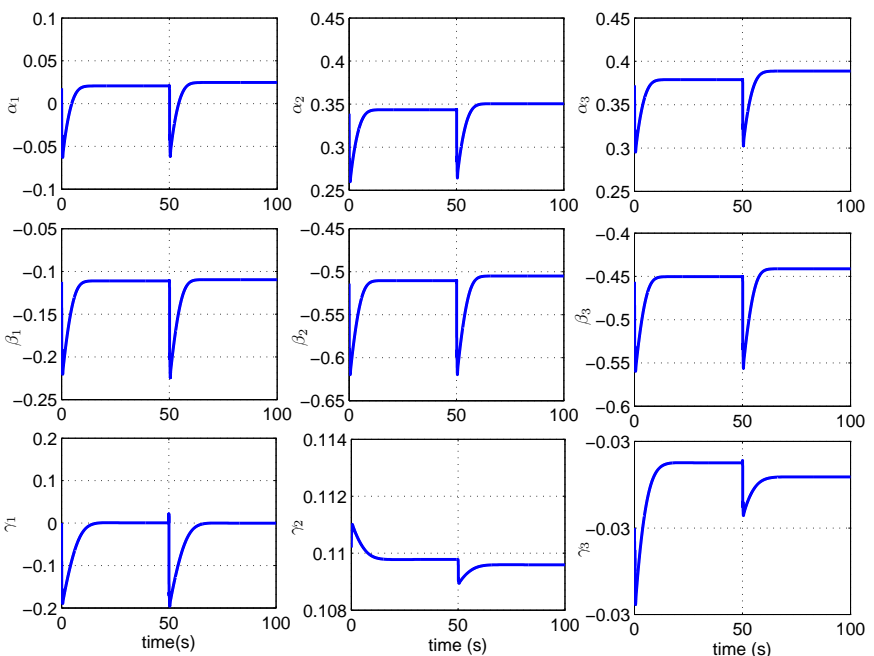

Fig. 9. Identification parameters of HNN.

effectiveness of the designed control scheme, the adaptive generalized predictive control (AGPC) proposed in [3], PID controller with HESS and the LFC without HESS are used to compare with the controller proposed in this paper. Fig. 8 shows the identification results of HNN and output of the PIDNN. Fig. 9 shows the identification of parameters $\hat{\alpha}_{i}, \hat{\beta}_{j}$ and $\hat{\gamma}_{l}$ of HNN.

From the simulation results, it is clear to see that the control effect of the frequency and tie-power deviations with HESS by the proposed controller is better than that of the PID controller, AGPC with HESS and the LFC without HESS. When load disturbances occur in a region, the frequency and tie-power deviations can be restored more quickly and smoothly by use of the proposed controller. Meanwhile, the HESS can operate within its constraints and reduce the power oscillations quickly and effectively by the proposed controller. The HNN can identify the system output effectively and the parameters of HNN can be adaptively adjusted during the running of the system.

\section{CONCLUSIONS}

Two neural networks have been used to design an identifier and controller in this paper. An adaptive PIDNN controller based on HNN has been developed for an improved fourarea interconnected LFC with the HESS. In the proposed control scheme, HNN is used as a forward identifier, which provides dynamic Jacobian information of four-area interconnected power system in real time to facilitate the design of adaptive control of PIDNN controller. Then, the PIDNN controller supplies the desired and appropriate power flow reference for the HESS. In addition, considering the capacity of HESS, a dynamic anti-windup signal is designed to solve the operational constraints of HESS. The stability of the whole system is analyzed by the Lyapunov theory. The simulation results demonstrate the effectiveness of the proposed control scheme, which tremendously reduces the frequency and tiepower deviations when compared with the PID controller with HESS and the LFC without HESS.

\section{ACKNOWLEDGMENT}

Our deepest gratitude goes to the editor and the anonymous reviewers for their careful work and thoughtful suggestions that have helped improve this paper substantially.

\section{REFERENCES}

[1] M. Mufti, S. Lone, S. Iqbal, et al. "Super-capacitor based energy storage system for improved load frequency control". Electric Power Systems Research, 2009, 79(1): 226-233.

[2] T. Tsay. "Load-frequency control of interconnected power system with governor backlash nonlinearities". International Journal of Electrical Power \& Energy Systems, 2011, 33(9): 1542-1549.

[3] M. Mufti, S. Iqbal, S. Lone, et al. "Supervisory adaptive predictive control scheme for supercapacitor energy storage system". IEEE Systems Journal, 2015, 9(3): 1-11.

[4] L. Dong, Y. Tang, H. He, et al. "An event-triggered approach for load frequency control with supplementary ADP”. IEEE Trans. on Power Systems, 2017, 32(1): 581-589.

[5] V. Singh, N. Kishor, P. Samuel. "Load frequency control with communication topology changes in smart grid". IEEE Trans. on Industrial Informatics, 2016, 12(5): 1943-1952.

[6] T. N. Pham, H. Trinh, V. H. Le, et al. "Integration of electric vehicles for load frequency output feedback $H_{\infty}$ control of smart grids". IET Generation Transmission \& Distribution, 2016, 10(13): 3341-3352.

[7] X. Liu, X. Kong, K. Lee. "Distributed model predictive control for load frequency control with dynamic fuzzy valve position modeling for hydro-thermal power system". IET Control Theory \& Applications, 2016, 10(14): 1653-1664.

[8] F. Liu, Y. Li, Y. Cao, et al. "A two-layer active disturbance rejection controller design for load frequency control of interconnected power system". IEEE Transactions on Power Systems, 2016, 31(4): 3320-3321.

[9] J. Pahasa, I. Ngamroo. "Coordinated control of wind turbine blade pitch angle and PHEVs using MPCs for load frequency control of microgrid". IEEE Systems Journal, 2016, 10(1): 97-105.

[10] R. Shankar, K. Chatterjee, R. Bhushan. "Impact of energy storage system on load frequency control for diverse sources of interconnected power system in deregulated power environment". International Journal of Electrical Power \& Energy Systems, 2016, 79: 11-26.

[11] R. Shankar, R. Bhushan, K. Chatterjee. "Small-signal stability analysis for two-area interconnected power system with load frequency controller in coordination with FACTS and energy storage device". Ain Shams Engineering Journal, 2015, 36(6): 41-49.

[12] R. K. Selvaraju, G. Somaskandan. "Impact of energy storage units on load frequency control of deregulated power systems". Energy, 2016, 97: 214-228.

[13] J. Li, Q. Yang, P. Yao, et al. "A novel use of the hybrid energy storage system for primary frequency control in a microgrid". Energy Procedia, 2016, 103: 82-87.

[14] N. J. V. Kumar, M. M. T. Ansari. "A new design of dual-mode Type-II fuzzy logic load frequency controller for interconnected power systems with parallel AC-DC tie-lines and superconducting magnetic energy storage unit". International Journal of Applied Engineering Research, 2015, 10(4): 9059-9084.

[15] M. G. Molina, P. E. Mercado. "Modeling of a DSTATCOM with ultracapacitor energy storage for power distribution system applications". Proc. 13th Eriac Decimo Tercer Encuentro Regional Iberoamericano De Cigre, 2009: 1-8.

[16] A. B. T. Attya, T. Hartkopf. "Utilising stored wind energy by hydropumped storage to provide frequency support at high levels of wind energy penetration". Iet Generation Transmission \& Distribution, 2015, 9(12): 1485-1497.

[17] S. Gurumurthy, V. Agarwal, A. Sharma. "High-efficiency bidirectional converter for flywheel energy storage application". IEEE Transactions on Industrial Electronics, 2016, 63(9): 1-1.

[18] D. Xu, F. Liu, B. Jiang. "Improved data driven model free adaptive constrained control for a solid oxide fuel cell". IET Control Theory \& Applications, 2016, 10(12): 1412-1419.

[19] N. Ji, D. Xu, F. Liu. "A novel adaptive neural network constrained control for solid oxide fuel cells via dynamic anti-windup". Neurocomputing, 2016, 214: 134-142.

[20] J. Liu, W. Luo, X. Yang, L. Wu, "Robust model-based fault diagnosis for PEM fuel cell air-feed system". IEEE Trans. on Industrial Electronics, 2016. 63(5): 3261-3270. 
[21] S. Sikkabut, P. Mungporn, C. Ekkaravarodome, et al. "Control of highenergy high-power densities storage devices by li-ion battery and supercapacitor for fuel cell/photovoltaic hybrid power plant for autonomous system applications". IEEE Transactions on Industry Applications, 2016, 52(5): 4395-4407.

[22] G. Dotelli, R. Ferrero, P. G. Stampino, et al. "Supercapacitor sizing for fast power dips in a hybrid supercapacitor-PEM fuel cell system". IEEE Transactions on Instrumentation \& Measurement, 2016, 65(10): 1-8.

[23] C. Attaianese, M. D. Monaco, G. Tomasso. "Power control for fuel-cellsupercapacitor traction drive". IEEE Transactions on Vehicular Technology, 2012, 61(5): 1961-1971.

[24] S. J. Iqbal, M. D. Mufti, S. A. Lone, et al. "Intelligently controlled superconducting magnetic energy storage for improved load frequency control". International Journal of Power \& Energy Systems, 2009, 29(4): 241.

[25] W. Li, G. Jos, J. Blanger. "Real-time simulation of a wind turbine generator coupled with a battery supercapacitor energy storage system". IEEE Transactions on Industrial Electronics, 2010, 57(4): 1137-1145.

[26] C. Yang, L. Zhang, J. Sun. "Anti-windup controller design for singularly perturbed systems subject to actuator saturation". IET Control Theory \& Applications, 2016, 10(4): 469-476.

[27] S. Vazquez, J. Rodriguez, M. Rivera, et al. "Model predictive control for power converters and drives: advances and trends". IEEE Transactions on Industrial Electronics, 2017, 64(2): 935-947.

[28] A. Lekka, M. C. Turner, P. P. Menon. "Anti-windup for a class of partially linearisable non-linear systems with application to wave energy converter control". IET Control Theory \& Applications, 2016, 10(18): 2403-2414

[29] W. Yan, J. Huang, D. Xu. "Adaptive command-filtered cackstepping control for linear induction motor via projection algorithm". Mathematical Problems in Engineering, 2016, 2016: 1-13.

[30] J. Liu, S. Vazquez, L. Wu, et al. "Extended state observer based sliding mode control for three-phase power converters". IEEE Transactions on Industrial Electronics, 2017, 64(1): 22-31.

[31] Y. Lin, A. Abur. "Highly efficient implementation for parameter error identification method exploiting sparsity". IEEE Transactions on Power Systems, 2017, 31(1): 734-742.

[32] J. Peng, R. Dubay. "Identification and adaptive neural network control of a DC motor system with dead-zone characteristics". ISA transactions, 2011, 50(4): 588-598.

[33] J. Michalkiewicz. "Modified Kolmogorov's neural network in the identification of Hammerstein and Wiener systems". IEEE Transactions on Neural Networks \& Learning Systems, 2012, 23(4): 657-662.

[34] S. Li, Y. Li. "Model predictive control of an intensified continuous reactor using a neural network Wiener model". Neurocomputing, 2015, 185: 4101-4106.

[35] M. Pouliquen, E. Pigeon, O. Gehan. "Identification Scheme for Hammerstein Output Error Models With Bounded Noise". IEEE Transactions on Automatic Control, 2016, 61(2): 550-555.

[36] F. Alonge, R. Rabbeni, M. Pucci, et al. "Identification and robust control of a quadratic DC/DC boost converter by Hammerstein model". IEEE Transactions on Industry Applications, 2015, 51(5): 3975-3985.

[37] X. Gao, X. Ren, C. Zhu, et al. "Identification and control for Hammerstein systems with hysteresis non-linearity". IET Control Theory \& Applications, 2015, 9(13): 1935-1947.

[38] W. Tan. "Unified tuning of PID load frequency controller for power systems via IMC". Energy Conversion \& Management, 2010, 25(1): 341350.

[39] J. Kang, W. Meng, A. Abraham, et al. "An adaptive PID neural network for complex nonlinear system control". Neurocomputing, 2014, 135(8): 79-85.

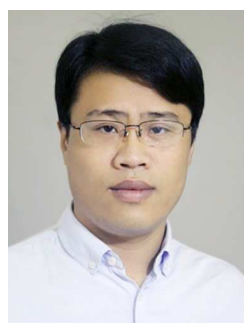

Dezhi Xu (M'15) received the Ph.D. degree in control theory and control engineering from the Nanjing University of Aeronautics and Astronautics, Nanjing, China, 2013. He is the committee member of the Association of Energy Internet in CAA. He joined Jiangnan University as the associate professor and master tutor. Now he is the reviewer for some international journals, such as several IEEE Trans. His research results as the important components received the 1st class award of science \& technology progression for China General Chamber of Commerce in 2016. He mainly researches on data-driven control, fault diagnosis and fault-tolerant control, technologies of new energy and power grid.

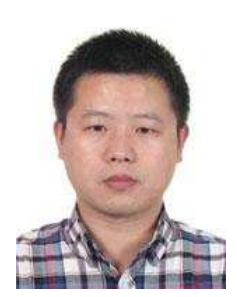

Jianxing Liu (M'13) received the B.S. degree in mechanical engineering and the M.E. degree in control science and engineering from the Harbin Institute of Technology, Harbin, China, in 2004 and 2010, respectively, and the Ph.D. degree in automation from the Technical University of BelfortMontbeliard, Belfort, France, in 2014. Since 2014, he has been with the Harbin Institute of Technology. His current research interests include sliding-mode control, nonlinear control and observation, industrial electronics, and renewable energy solutions.

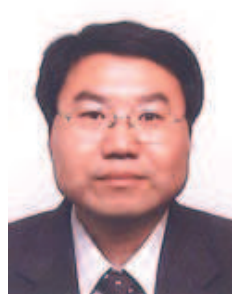

Xing-Gang Yan received the B.Sc. degree from Shaanxi Normal University, Xi'an, China, in 1985, the M.Sc. degree from Qufu Normal University, Jining, China, in 1991, and the Ph.D. degree in engineering from Northeastern Uni- versity, Shenyang, China, in 1997. He was a Lecturer with Qingdao University, China, from 1991 to 1994 . He was a Research Fellow/Associate with the University of Hong Kong, Hong Kong, with Nanyang Technological University, Singapore, and with the University of Leicester, U.K. He is currently a Senior Lecturer with the University of Kent, Canterbury, U.K. His research interests include sliding mode control, decentralized control, fault detection and isolation, and control and observation of nonlinear systems and time-delay systems with applications.

Dr. Yan is the Editor-in-Chief of the International Journal of Engineering Research and Science and Technology.

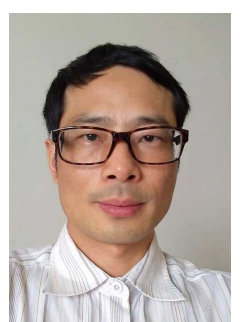

Wenxu Yan received the Ph.D. degree in control theory and control engineering from the Jiangnan University, Wuxi, China. He is currently an associate professor and master tutor with the Institute of Automation, Jiangnan University. He has published more than 30 academic papers in domestic and international journals or conferences. His current research interests include intelligent control, industrial electronics, smart grid, and renewable energy solutions. 